\title{
A NOTE ON SOLUTIONS OF YAMABE-TYPE EQUATIONS ON PRODUCTS OF SPHERES
}

\author{
JIMMY PETEAN AND HÉCTOR BARRANTES G.
}

\begin{abstract}
We consider Yamabe-type equations on the Riemannian product of constant curvature metrics on $\mathbf{S}^{n} \times \mathbf{S}^{n}$, and study solutions which are invariant by the cohomogeneity one diagonal action of $O(n+1)$. We obtain multiplicity results for both positive and nodal solutions. In particular we prove the existence of nodal solutions of the Yamabe equation on these products which depend non-trivially on both factors.
\end{abstract}

\section{INTRODUCTION}

Given a Riemannian manifold $\left(M^{n}, g\right)$ of dimension $n \geq 3$, the Yamabe equation is

$$
-a_{n} \Delta_{g} u+s_{g} u=\lambda|u|^{p_{n}-2} u,
$$

where $s_{g}$ is the scalar curvature of $g, a_{n}=\frac{4(n-1)}{n-2}, p_{n}=\frac{2 n}{n-2}$ and $\lambda \in \mathbb{R}$. In case $u$ is a positive solution of this equation then the conformal metric $u^{p_{n}-2} g$ has constant scalar curvature $\lambda$. An important role in the study of the equation has been played by the Yamabe constant of the conformal class $[g]$ of $g$, which we denote by $Y(M,[g])$ and is given by

$$
Y(M,[g])=\inf _{h \in[g]} \frac{\int_{M} s_{h} d v_{h}}{\left(\operatorname{Vol}(M, h)^{\frac{n-2}{n}}\right.},
$$

where $d v_{h}$ is the volume element of the metric $h$ and $\operatorname{Vol}(M, h)$ is the volume of $(M, h)$. Throughout the combined efforts of H. Yamabe [29], N. Trudinger [28, T. Aubin [5] and R. Schoen [25] it was proved that the equation always has at least one solution, for which the corresponding constant scalar curvature metric realizes the Yamabe constant. The equation can be normalized so that $\lambda$ is $-1,0$ or 1 , according to the sign of $Y(M, g)$. The minimizing solution is the unique solution in case $Y(M,[g]) \leq 0$, i.e. $\lambda=-1$ or $\lambda=0$. It is also unique in case the minimizing solution is Einstein and different from the constant curvature metric on $\mathbf{S}^{n}$, which we will denote by $g_{0}^{n}$, by a theorem of M. Obata [22]. $\left(\mathbf{S}^{n}, g_{0}^{n}\right)$ has a non-compact group of conformal transformations, which give a non-compact family of solutions to the Yamabe equation. This is the first example of multiplicity of solutions and plays an important role in the theory. Another important example, or family of examples, where one has multiplicity of solutions is that of Riemannian products. If

J. Petean is supported by grant 220074 of Fondo Sectorial de Investigación para la Educación SEP-CONACYT. 
$(M \times N, g+h)$ is a Riemannian product with constant scalar curvature, with $s_{h}>0$, then one considers $\left(M^{n} \times N^{k}, g+\delta h\right)$ for $\delta>0$ and small. It can be seen by general considerations that for $\delta$ small enough the product metric cannot be a minimizer for the Yamabe constant. Therefore there must be at least one other solution. But there are several results showing that the number of solutions grows as $\delta \rightarrow 0$, see for instance [6, 12, 17, 26]. The solutions built in these articles are actually functions of $M$. A function $u: M \rightarrow \mathbb{R}$, considered as a function on $M \times N$ gives a solution for the Yamabe equation for $g+\delta h$ if it satisfies

$$
-a_{n+k} \Delta_{g} u+\left(s_{g}+\delta^{-1} s_{h}\right) u=|u|^{p_{n+k}-2} u,
$$

where we have normalized the positive constant $\lambda$ to be 1 . Note that $p_{n+k}<p_{n}$. So in this context one would be interested in positive solutions of the subcritical equation

$$
-\Delta u+\lambda u=\lambda|u|^{p-2} u
$$

where $\lambda$ is a positive constant, $2<p \leq p_{n}$, and we have now renormalized the equation so that $u \equiv 1$ is a solution.

Many multiplicity results have also been obtained for these equations using LyapunovSchmidt reduction and other topological methods (see for instance [10, 13, 20]).

There has also been interest in nodal solutions of the equations (i.e. solutions that change sign). See for instance the articles [4, 8, 9, 14, 15, 16, 24] and the references in them. Nodal solutions $u$ do not give metrics of constant scalar curvature since $u$ vanishes at some points and therefore $|u|^{p_{n}-2} g$ is not a Riemannian metric. But they have geometric interest. The existence of at least one nodal solution is proved in general cases in [4], as minimizers for the second Yamabe invariant. But there are not as many results about multiplicity of nodal solutions as in the positive case.

In this article we will consider the products of spheres $\left(\mathbf{S}^{n} \times \mathbf{S}^{n}, g_{0}^{n} \times \delta g_{0}^{n}\right)$, with $\delta>0$ (note that for $\delta=1$ the metric is Einstein). Solutions for $\delta$ small and subcritical exponent have been built in [17, 19, 23], which depend only on the first factor. Interest in finding all solutions of the Yamabe equation in this case comes from trying to compute the Yamabe constants and its $\operatorname{limit}_{\delta \rightarrow 0} Y\left(\mathbf{S}^{n} \times \mathbf{S}^{n},\left[g_{0}^{n}+\delta g_{0}^{n}\right]\right)=Y\left(\mathbf{S}^{n} \times \mathbb{R}^{n}, g_{0}^{n}+d x^{2}\right)$ (see [2]). An important question raised in [2, 3], related to the computations of these Yamabe constants, is whether all solutions of the Yamabe equation on certain Riemannian products, like products of spheres (or the product of a sphere with Euclidean space), depend on only one of the factors. The main goal of this article is to show the existence of solutions which depend non-trivially on both factors: positive solutions when $p<p_{n}$ and nodal solutions when $p=p_{n}$. To build such solutions we consider the isometric $O(n+1)$-action on $\mathbf{S}^{n} \times \mathbf{S}^{n}$ given by $A \cdot(x, y)=(A x, A y)$. It is a cohomogeneity one action. From now on by an invariant function on $\mathbf{S}^{n} \times \mathbf{S}^{n}$ we will mean a function which is invariant by this diagonal $O(n+1)$-action, there is no risk of confusion since we will only consider this action. Note that any invariant function which is not constant depends non-trivially on both factors of $\mathbf{S}^{n} \times \mathbf{S}^{n}$. 
We first consider nodal solutions of the (critical) Yamabe equation on the products $\left(\mathbf{S}^{n} \times \mathbf{S}^{n}, g_{0}^{n}+\delta g_{0}^{n}\right)$. We will prove:

Theorem 1.1. For any $\delta>0$ the Yamabe equation on $\left(\mathbf{S}^{n} \times \mathbf{S}^{n}, g_{0}^{n}+\delta g_{0}^{n}\right)$,

$$
-a_{2 n} \Delta_{g_{0}^{n}+\delta g_{0}^{n}} u+n(n-1)\left(1+\delta^{-1}\right) u=n(n-1)\left(1+\delta^{-1}\right)|u|^{p_{2 n}-2} u
$$

admits infinite nodal solutions which are invariant by the diagonal action of $O(n+1)$.

We will also prove that the number of positve solutions of the subcritical equation grows as $\lambda \rightarrow \infty$. As we mentioned before this gives multiplicity results for the Yamabe equation on certain Riemannian products. We will prove:

Theorem 1.2. For each $\delta>0, p \in\left(2, p_{2 n}\right)$, let $\lambda_{k, \delta, p}:=\frac{k(k+n-1)}{p-2}\left(1+\frac{1}{\delta}\right)$. If $\lambda \in$ $\left(\lambda_{k, \delta, p}, \lambda_{k+1, \delta, p}\right]$ then the subcritical equation on $\left(\mathbf{S}^{n} \times \mathbf{S}^{n}, g_{0}^{n} \times \delta g_{0}^{n}\right)$,

$$
-\Delta_{g_{0}^{n}+\delta g_{0}^{n}} u+\lambda u=\lambda u^{p-1},
$$

has at least $k$ positive solutions which are invariant by the diagonal action of $O(n+1)$.

In Section 2 we will discuss the setting of Yamabe-type equations restricted to the space of functions invariant by the diagonal action of $O(n+1)$ on $\mathbf{S}^{n} \times \mathbf{S}^{n}$. We will discuss nodal solutions of the Yamabe equation and prove Thoerem 1.1 in Section 3. Finally in Section 4 we will consider positive solutions of subcritical equations and prove Theorem 1.2.

\section{YAMABE-TYPE EQUATIONS FOR INVARIANT FUNCTIONS}

We consider $n \geq 2$ and let $g_{0}^{n}$ denote the curvature 1 metric on $\mathbf{S}^{n}$. For any $\delta>0$ we consider the Riemannian product $G_{\delta}=g_{0}^{n}+\delta g_{0}^{n}$ and the isometric action of $O(n+1)$ on $\left(\mathbf{S}^{n} \times \mathbf{S}^{n}, G_{\delta}\right)$ given by $A \cdot(x, y)=(A x, A y)$.

Let $f: \mathbf{S}^{n} \times \mathbf{S}^{n} \rightarrow[-1,1]$ be given by:

$$
f(p, q)=\langle p, q\rangle .
$$

Note that $f$ is invariant by the action of $O(n+1)$. By a direct computation we obtain

$$
\Delta_{G_{\delta}} f=-n\left(1+\frac{1}{\delta}\right) f, \quad\left|\nabla_{G_{\delta}} f\right|_{G_{\delta}}^{2}=\left(1+\frac{1}{\delta}\right)\left(1-f^{2}\right) .
$$

This implies that $f$ is an isoparametric function (see [30] for the definition and basic results concerning isoparametric functions). The only critical values of $f$ are its minimum -1 and its maximum 1. Every invariant function $u: \mathbf{S}^{n} \times \mathbf{S}^{n} \rightarrow \mathbb{R}$ can be written as $u=\varphi \circ f$, where $\varphi:[-1,1] \rightarrow \mathbb{R}$. Since $f$ is smooth the regularity of $u$ is equal to the regularity of $\varphi$. We have that

$$
\Delta_{G_{\delta}} u=\left(\varphi^{\prime \prime} \circ f\right) \cdot\left|\nabla_{G_{\delta}} f\right|_{G_{\delta}}^{2}+\left(\varphi^{\prime} \circ f\right) \cdot \Delta_{G_{\delta}} f
$$




$$
=\left[\left(1+\frac{1}{\delta}\right)\left(1-t^{2}\right) \varphi^{\prime \prime}-n\left(1+\frac{1}{\delta}\right) t \varphi^{\prime}\right] \circ f
$$

for $t \in[-1,1]$.

Therefore $u$ solves

$$
-\Delta_{G_{\delta}} u+\lambda u=\lambda|u|^{p-2} u \text { on } \mathbf{S}^{n} \times \mathbf{S}^{n}
$$

if and only if

$$
-\left(1-t^{2}\right) \varphi^{\prime \prime}(t)+n t \varphi^{\prime}(t)+\frac{\lambda}{1+\frac{1}{\delta}} \varphi(t)=\frac{\lambda}{1+\frac{1}{\delta}}|\varphi|^{p-2} \varphi .
$$

If we now call $w(r)=\varphi(\cos (r))$ then $w^{\prime}(0)=w^{\prime}(\pi)=0$ and $\varphi$ solves equation (44) if and only if

$$
w^{\prime \prime}(r)+(n-1) \frac{\cos (r)}{\sin (r)} w^{\prime}(r)+\frac{\lambda}{1+\frac{1}{\delta}}\left(|w(r)|^{p-2} w(r)-w(r)\right)=0
$$

with $r \in[0, \pi]$.

For any $\alpha>0$ we call $w_{\alpha}:[0, \pi) \rightarrow \mathbb{R}$ the solution of (5) with initial conditions

$$
w_{\alpha}(0)=\alpha, \quad w_{\alpha}^{\prime}(0)=0 .
$$

If $w_{\alpha}$ extends up to the singularity at $\pi$ and $w_{\alpha}^{\prime}(\pi)=0$, then $\varphi_{\alpha}(t)=w_{\alpha}(\arccos (t))$ is a $C^{2}$ function which solves equation (44). Then $u=\varphi_{\alpha} \circ f$ solves equation (3).

When $\lambda=\frac{s_{G_{\delta}}}{a_{2 n}}=\frac{n(n-1)(2 n-2)\left(1+\frac{1}{\delta}\right)}{4(2 n-1)}$ and $p=\frac{4 n}{2 n-2}$ equation (3) is the Yamabe equation for $\left(\mathbf{S}^{n} \times \mathbf{S}^{n}, G_{\delta}\right)$. Therefore Theorem 1.1 follows from the following:

Theorem 2.1. Let $p=\frac{4 n}{2 n-2}$. For any $\lambda>0$ and any integer $k$ there exists $\alpha_{k}>0$ such that $w_{\alpha_{k}}^{\prime}(\pi)=0$ and $w_{\alpha_{k}}$ has exactly $k$ zeroes on $(0, \pi)$.

Similarly Theorem 1.2 follows from the next theorem. For any $\delta>0$ and any $p \in\left(2, p_{2 n}\right)$ we call $\lambda_{k}=\frac{k(k+n-1)}{p-2}\left(1+\delta^{-1}\right)$. Then we have:

Theorem 2.2. For any $p \in\left(2, p_{2 n}\right), \delta>0$ and $\lambda \in\left(\lambda_{k}, \lambda_{k+1}\right]$ there exist at least $k$ positive different solutions of equation (5) verifying the boundary conditions $w^{\prime}(0)=$ $w^{\prime}(\pi)=0$.

Theorem 2.1 will be proved in Section 3 and Theorem 2.2 will be proved in Section 4. To finish this section we introduce the energy functional. Let $\mu=\frac{\lambda}{1+\frac{1}{\delta}}$ and

$$
E_{\alpha}(r):=\frac{1}{2}\left(w_{\alpha}^{\prime}(r)\right)^{2}+\mu\left(\frac{\left|w_{\alpha}(r)\right|^{p}}{p}-\frac{w_{\alpha}^{2}(r)}{2}\right)
$$

Note that 


$$
E_{\alpha}^{\prime}(r)=-(n-1) \frac{\cos (r)}{\sin (r)}\left(w_{\alpha}^{\prime}(t)\right)^{2}
$$

therefore $E_{\alpha}$ is decreasing in $(0, \pi / 2)$ and increasing in $(\pi / 2, \pi)$.

Note also that if $w_{\alpha}\left(r_{0}\right)=0$ then $E_{\alpha}\left(r_{0}\right)=\frac{1}{2}\left(w_{\alpha}^{\prime}\left(r_{0}\right)\right)^{2} \geq 0$ (and the equality holds if and only if $\alpha=0)$. For instance this implies

Lemma 2.3. If $\alpha>0$ is such that $E_{\alpha}(0) \leq 0$ then $w_{\alpha}(r)>0$, for all $r \in\left(0, \frac{\pi}{2}\right)$.

Note that

$$
E_{\alpha}(0)=\frac{1}{2}\left(w_{\alpha}^{\prime}(0)\right)^{2}+\mu\left(\frac{\left|w_{\alpha}(0)\right|^{p}}{p}-\frac{w_{\alpha}^{2}(0)}{2}\right)=\mu\left(\frac{\alpha^{p}}{p}-\frac{\alpha^{2}}{2}\right)=\mu \frac{\alpha^{2}}{p}\left(\alpha^{p-2}-\frac{p}{2}\right)
$$

and the lemma says that if $w_{\alpha}$ has a zero in $\left(0, \frac{\pi}{2}\right)$, then $\alpha>\left(\frac{p}{2}\right)^{\frac{1}{p-2}}$.

\section{Proof of Theorem 2.1}

We fix $p=p_{2 n}$ in equation (5). We begin with some elementary lemmas concerning equation (5). We call $\mu=\frac{\lambda}{1+\frac{1}{\delta}}$.

Lemma 3.1. Let $\alpha>0$ be such that the solution $w_{\alpha}$ of (5) satisfies $w_{\alpha}^{\prime}\left(\frac{\pi}{2}\right)=0$ then $w_{\alpha}(\pi-t)=w_{\alpha}(t)$ for all $t \in[0, \pi)$ and therefore $w_{\alpha}^{\prime}(\pi)=0$.

Proof. Let $h(t)=w_{\alpha}(\pi-t)$ with $t \in[0, \pi)$. Note that $h$ is a solution of equation (5). Moreover $h\left(\frac{\pi}{2}\right)=w_{\alpha}\left(\frac{\pi}{2}\right), h^{\prime}\left(\frac{\pi}{2}\right)=w_{\alpha}^{\prime}\left(\frac{\pi}{2}\right)=0$. By the uniqueness of solutions $h=w_{\alpha}$. Therefore $w_{\alpha}(\pi-t)=h(t)=w_{\alpha}(t)$, proving the lemma.

Lemma 3.2. Let $\alpha>0$ be such that the solution $w_{\alpha}$ de (5) satisfies $w_{\alpha}\left(\frac{\pi}{2}\right)=0$. Then $w_{\alpha}(\pi-t)=-w_{\alpha}(t)$ for all $t \in[0, \pi)$ and therefore $w_{\alpha}^{\prime}(\pi)=0$.

Proof. Let $h(t)=-w_{\alpha}(\pi-t)$ with $t \in[0, \pi)$. Note that $h$ is a solution of equation (5). Since $h\left(\frac{\pi}{2}\right)=w_{\alpha}\left(\frac{\pi}{2}\right)=0, h^{\prime}\left(\frac{\pi}{2}\right)=w_{\alpha}^{\prime}\left(\frac{\pi}{2}\right)$ it follows from the uniqueness of solutions that $h=w_{\alpha}$, proving the lemma.

Lemma 3.3. Let $\alpha_{0}>1$ be such that the solution $w_{\alpha_{0}}$ of (5) has exactly $k$ zeroes in $\left(0, \frac{\pi}{2}\right)$ and $w_{\alpha_{0}}\left(\frac{\pi}{2}\right) \neq 0$. Then there exists $\varepsilon>0$ such that for any $\alpha \in\left(\alpha_{0}-\varepsilon, \alpha_{0}+\varepsilon\right)$ the solution $w_{\alpha}$ has exactly $k$ zeroes in $\left(0, \frac{\pi}{2}\right)$ and $w_{\alpha}(\pi / 2) \neq 0$.

Proof. Let $0<z_{1}<\ldots, z_{k}<\pi / 2$ be the $\mathrm{k}$ zeroes of $w_{\alpha_{0}}$ in $(0, \pi / 2)$. Let $\delta>0$ be small enough so that $w_{\alpha_{0}}^{\prime}(t) \neq 0$ for any $i=1, \ldots, k$ and any $t \in\left[z_{i}-\delta, z_{i}+\delta\right]$. For $\varepsilon>0$ small enough we can assume that for any $\alpha \in\left(\alpha_{0}-\varepsilon, \alpha_{0}+\varepsilon\right)$ we have that $w_{\alpha}>0$ in $\left[0, z_{1}-\delta\right], w_{\alpha}^{\prime}<0$ in $\left[z_{1}-\delta, z_{1}+\delta\right], w_{\alpha}<0$ in $\left[z_{1}+\delta, z_{2}-\delta\right], w_{\alpha}^{\prime}>0$ in $\left[z_{2}-\delta, z_{2}+\delta\right]$, and so on. It follows that $w_{\alpha}$ has exactly $k$ zeroes in $(0, \pi / 2)$.

Lemma 3.4. Let $\alpha_{0}>1$ be such that the solution $w_{\alpha_{0}}$ of equation (5) has exactly $k$ zeroes in $\left(0, \frac{\pi}{2}\right)$ and $w_{\alpha_{0}}\left(\frac{\pi}{2}\right)=0$. Then there exists $\varepsilon>0$ such that for any $\alpha \in\left(\alpha_{0}-\varepsilon, \alpha_{0}+\varepsilon\right)$ the solution $w_{\alpha}$ has either exactly $k$ zeroes or exactly $k+1$ zeroes in $\left(0, \frac{\pi}{2}\right)$. 
Proof. Since $w_{\alpha_{0}}\left(\frac{\pi}{2}\right)=0$ and $\alpha_{0} \neq 0$ we have that $w_{\alpha_{0}}^{\prime}\left(\frac{\pi}{2}\right) \neq 0$. Let $\delta>0$ be small enough so that $w_{\alpha_{0}}^{\prime}(t) \neq 0$ if $t \in[\pi / 2-\delta, \pi / 2+\delta]$. Choose $\varepsilon>0$ small enough so that for any $\alpha \in\left(\alpha_{0}-\varepsilon, \alpha_{0}+\varepsilon\right) w_{\alpha}^{\prime}(t) \neq 0$ for any $t \in[\pi / 2-\delta, \pi / 2+\delta]$ and $w_{\alpha}$ has exactly one zero in $[\pi / 2-\delta, \pi / 2+\delta]$. By the same argument as in the previous lemma we can also assume that $\varepsilon$ is small enough so that for any $\alpha \in\left(\alpha_{0}-\varepsilon, \alpha_{0}+\varepsilon\right)$ the solution $w_{\alpha}$ has exactly $k$ zeroes in $[0, \pi / 2-\delta]$. Therefore $w_{\alpha}$ has either $k$ or $k+1$ in $(0, \pi / 2)$, depending on whether its zero in $[\pi / 2-\delta, \pi / 2+\delta]$ is $<\pi / 2$ or not.

Now we are ready to prove Theorem 2.1.

Proof. Note that we are considering equation (5) with $p=p_{2 n}<p_{n}$. Consider an integer $i>>$. It then follows from [15, Theorem 3.1] that there exists $\alpha_{*}>1$ such that $w_{\alpha_{*}}$ has at least $i$ zeroes in $\left(0, \frac{\pi}{2}\right)$.

First consider the set

$$
A_{0}:=\left\{\alpha \in\left(1, \alpha_{*}\right]: w_{\alpha} \geq 0 \text { in }\left(0, \frac{\pi}{2}\right]\right\} .
$$

Note that by Lemma $2.3\left(1,\left(\frac{p}{2}\right)^{\frac{1}{p-2}}\right] \subset A_{0}$ and that $A_{0}$ is closed in $\left(1, \alpha_{*}\right]$. Let $a_{0}:=\sup A_{0}$. If $t \in(0, \pi / 2)$ and $w_{a_{0}}(t)=0$ then $t$ would be a local minimum for $w_{a_{0}}$ and therefore $w_{a_{0}}^{\prime}(t)=0$. By uniqueness we would have that $w_{a_{0}} \equiv 0$, which is a contradiction. Then $w_{a_{0}}$ is strictly positive in $[0, \pi / 2)$. Moreover $w_{a_{0}}\left(\frac{\pi}{2}\right)=0$, since $a_{0}$ is the supremum of $A_{0}$. Therefore by Lemma $3.2 w_{a_{0}}^{\prime}(\pi)=0$ and $w_{a_{0}}$ has exactly one zero in $[0, \pi]$.

Now define

$$
A_{1}:=\left\{\alpha \in\left(1, \alpha_{*}\right]: w_{\alpha} \text { has exactly one zero in }\left(0, \frac{\pi}{2}\right)\right\}
$$

If $a_{0}<\alpha \leq \alpha_{*}$ then $w_{\alpha}$ has at least one zero in $\left(0, \frac{\pi}{2}\right)$. Therefore by Lemma 3.4 there exists $a>a_{0}$ such that $w_{a}$ has exactly one zero in $\left(0, \frac{\pi}{2}\right)$. Therefore $A_{1} \neq \emptyset$ and it is bounded. Let $a_{1}:=\sup A_{1}$. Note that $a_{1}>a_{0}$. By Lemma $3.3 w_{a_{1}}(\pi / 2)=0$. Since $a_{1}>a_{0}$ it follows that $w_{a_{1}}$ has exactly one zero in $\left(0, \frac{\pi}{2}\right)$.

Now for any $j \geq 2, j<i$ define

$$
A_{j}:=\left\{\alpha \in\left(1, \alpha_{*}\right]: w_{\alpha} \text { has exactly j zeroes in }\left(0, \frac{\pi}{2}\right)\right\}
$$

Assume that $A_{j} \neq \emptyset$ and $a_{j}=\sup A_{j}>a_{j-1}>a_{j-2}>\ldots>a_{1}$. If $j+1<i$ then it follows from Lemma 3.3 and Lemma 3.4 that $w_{a_{j}}(\pi / 2)=0, A_{j+1} \neq \emptyset$. As in the case $j=1$ we see then that $a_{j+1}=\sup A_{j+1}>a_{j}$. By induction we see that $\forall j \geq 2, A_{j} \neq \emptyset$ and $a_{j}>a_{j-1}$. This implies that for any $0 \leq j<i$ there exists $a_{j}>1$ such that $w_{a_{j}}(\pi / 2)=0$ and $w_{a_{j}}$ has exactly $j$ zeroes in $(0, \pi / 2)$. Then by Lemma $3.2 w_{a_{j}}^{\prime}(\pi)=0$ and $w_{a_{j}}$ has exactly $2 j+1$ zeroes in $(0, \pi)$. This means that we have proved the theorem in case $k$ is odd.

On the other hand since $w_{a_{j}}$ has exactly one zero less than $w_{a_{j+1}}$ in $(0, \pi / 2)$, it follows that $w_{a_{j}}^{\prime}\left(\frac{\pi}{2}\right)$ and $w_{a_{j+1}}^{\prime}\left(\frac{\pi}{2}\right)$ have different signs. It then follows that there exists $a \in\left(a_{j}, a_{j+1}\right)$ such that $w_{a}^{\prime}(\pi / 2)=0$. Let $b_{j}=\inf \left\{a \in\left(a_{j}, a_{j+1}\right): w_{a}^{\prime}(\pi / 2)=0\right\}$. Then $w_{b_{j}}^{\prime}(\pi / 2)=0$ and for any $a \in\left(a_{j}, b_{j}\right)$ we have that $w_{a}^{\prime}(\pi / 2)$ has the same sign 
as $w_{a_{j}}^{\prime}\left(\frac{\pi}{2}\right)$. By the definition of $a_{j}$ (and the discussion above) we know that for any $a>a_{j} w_{a}$ has at least $j+1$ zeroes in $(0, \pi / 2)$ and if moreover $a$ is close to $a_{j}$ then $w_{a}$ has exactly $j+1$ zeroes. Since the sign of $w_{a}^{\prime}(\pi / 2)$ does not change it then follows that $w_{a}$ has exactly $j+1$ zeroes for all $a \in\left(a_{j}, b_{j}\right)$ and Lemma 3.3 implies that $w_{b_{j}}$ also has exactly $j+1$ zeroes in $(0, \pi / 2)$. Then by Lemma $3.1 w_{b_{j}}^{\prime}(\pi)=0$ and $w_{b_{j}}$ has exactly $2(j+1)$ zeroes in $(0, \pi)$. This proves the theorem when $k$ is even and we have therefore concluded the proof of the thoerem.

\section{Bifurcation for positive solutions, proof of Theorem 2.2}

We use bifurcation theory. We denote by $X_{I}$ the set of invariant functions on $\mathbf{S}^{n} \times \mathbf{S}^{n}$. Consider the Banach space

$$
C^{2, \alpha}\left(X_{I}\right):=X_{I} \cap C^{2, \alpha}\left(\mathbf{S}^{n} \times \mathbf{S}^{n}\right) .
$$

As in Section 2 we identify $C^{2, \alpha}\left(X_{I}\right)$ with the set of functions $w \in C^{2, \alpha}([0, \pi])$ such that $w^{\prime}(0)=w^{\prime}(\pi)=0$. Similarly we define $C^{0, \alpha}\left(X_{I}\right):=X_{I} \cap C^{0, \alpha}\left(\mathbf{S}^{n} \times \mathbf{S}^{n}\right)$, which is identified with $C^{0, \alpha}([0, \pi])$. Now let $C_{+}^{2, \alpha}\left(X_{I}\right)$ denote the set of positive functions on $C^{2, \alpha}\left(X_{I}\right)$.

We define $S: C_{+}^{2, \alpha}\left(X_{I}\right) \times \mathbb{R}_{\geq 0} \rightarrow C^{0, \alpha}\left(X_{I}\right)$ by

$$
S(u, \lambda)=-\Delta_{G_{\delta}} u+\lambda\left(u-u^{p-1}\right) .
$$

We have for any $\lambda \geq 0$ that $S(1, \lambda)=0$ and we will study solutions of $S(u, \lambda)=0$ which bifurcate for the curve $(1, \lambda)$. The local bifurcation theory is well known, any detail about what we will use in this section can be found for instance in Chapter 2 of [1] or in [21].

Note that

$$
S_{u}^{\prime}(1, \lambda)[v]=-\Delta_{G_{\delta}} v-\lambda(p-2) v .
$$

Then as in Section 2, we write $v(x)=w(\arccos f(x))$ for a function $w:[0, \pi] \rightarrow \mathbb{R}$ with $w^{\prime}(0)=w^{\prime}(\pi)=0$, and $S_{u}^{\prime}(1, \lambda)[v]=0$ if and only if

$$
w^{\prime \prime}(r)+(n-1) \frac{\cos (r)}{\sin (r)} w^{\prime}(r)+\frac{\lambda(p-2)}{1+\frac{1}{\delta}} w(r)=0
$$

We are then led to consider for any positive constant $\beta$ the solution $w_{\beta}$ of the initial value problem

$$
w^{\prime \prime}(r)+(n-1) \frac{\cos (r)}{\sin (r)} w^{\prime}(r)+\beta w(r)=0, \quad w(0)=1, \quad w^{\prime}(0)=0 .
$$

This equation is well-known, it corresponds to the eigenvalue equation for the Laplacian on the sphere. If $\beta_{k}=k(n+k-1)$ then $w_{\beta_{k}}$ can be computed explicitly. For instance: 
$w_{\beta_{1}}(r)=\cos (r), \quad w_{\beta_{2}}(r)=\frac{n+1}{n} \cos ^{2}(r)-\frac{1}{n}, \quad w_{\beta_{3}}(r)=\frac{n+4}{n-2} \cos ^{3}(r)-\frac{6}{n-2} \cos (r)$.

In general we call

$$
H_{\beta}(w):=w^{\prime \prime}(r)+(n-1) \frac{\cos (r)}{\sin (r)} w^{\prime}(r)+\beta w(r) .
$$

Then, for each positive integer $k$ we have

$$
H_{\beta}\left(\cos ^{k}(r)\right)=\left(\beta-\beta_{k}\right) \cos ^{k}(r)+k(k-1) \cos ^{k-2}(r) .
$$

It is then easy to see that $w_{\beta_{k}}(r)=p_{k}(\cos (r))$, where $p_{k}$ is a polynomial of degree $k$. If $k$ is odd then $p_{k}$ is a sum of monomials of odd degree and if $k$ is even $p_{k}$ is a sum of monomials of even degree.

For each positive integer $k$, note that $\lambda_{k}=\frac{\beta_{k}}{p-2}\left(1+\frac{1}{\delta}\right)$ and denote by $L_{k}=$ $S_{u}^{\prime}\left(1, \lambda_{k}\right)$. Then by the previous considerations $\operatorname{ker}\left(L_{k}\right)=\left\langle w_{\beta_{k}}\right\rangle$ has dimension 1 .

Note that by integration by parts if $x \in C^{2, \alpha}\left(X_{I}\right)$ then

$$
0=\int_{0}^{\pi} L_{k}\left(w_{\beta_{k}}\right) x d r=\int_{0}^{\pi} L_{k}(x) w_{\beta_{k}} d r .
$$

This implies that the range $R(L)$ of $L$, is

$$
R\left(L_{k}\right)=\left\{y \in C^{0, \alpha}([0, \pi]): \int_{0}^{\pi} y w_{k} d r=0\right\} .
$$

On the other hand, note that

$$
S_{u, \lambda}^{\prime \prime}\left(1, \lambda_{k}\right)\left[w_{\beta_{k}}\right]=(p-2) w_{\beta_{k}},
$$

and since $\int_{0}^{\pi} w_{k}^{2} d r \neq 0$ we have that

$$
S_{u, \lambda}^{\prime \prime}\left(1, \lambda_{k}\right)\left[w_{k}\right] \notin R\left(L_{k}\right)
$$

Therefore, by ([1, Theorem 2.8]), the points $\left(1, \lambda_{k}\right)$ are bifurcation points of $S(u, \lambda)=$ 0 . Moreover, close to $\left(1, \lambda_{k}\right)$ the space of solutions consists of two curves: one is the curve of trivial solutions $\lambda \mapsto(1, \lambda)$, and the other one is a curve of nontrivial solutions which has the form $t \mapsto(u(t), \lambda(t))$ where $\lambda(0)=\lambda_{k}$ and $u(t)=1+t w_{\beta_{k}}+o\left(t^{2}\right)$.

Note that if $u$ is a nontrivial solution then for any $r \in(0, \pi)$, if $u(r)=1$ then $u^{\prime}(r) \neq 0$. It follows that the number of zeroes of $u-1$ is constant in an open $C^{2}$-neighborhood of $u$.

Now, we point out that the solution $w_{\beta_{k}}$, which generates ker $L_{k}$, has exactly $k$ zeroes in $(0, \pi)$. This is explicitly proved for instance in [19] and in [23], we just give a sketch of a proof for completeness:

First note that since $p_{k}$ is a polynomial of degree $k$ then $w_{\beta_{k}}$ can have at most $k$ zeroes in $(0, \pi)$ and they must be simple since $w_{\beta_{k}}$ solves a second order linear ordinary differential equation. Then by direct calculation, we can verify that $w_{\beta_{i}}$, have $i$ zeroes in $(0, \pi)$, for $i=1,2,3$. Let $m<l$ be positive integers. Since $\beta_{m}=$ 
$m(n+m-1)<l(n+l-1)=\beta_{l}$ then, by the Sturm comparison theorem (see for instance [18, Page 229]), between any two zeroes of $w_{\beta_{m}}$ there is at least one zero of $w_{\beta_{l}}$. Hence, $w_{\beta_{l}}$ has at least the same number of zeroes as $w_{\beta_{m}}$ and if it has exactly the same number, then $w_{\beta_{l}}$ and $w_{\beta_{m}}$ have the same sign after the last zero. Suppose that $w_{k}$ has $k$ zeroes in $(0, \pi)$. Notice that if $k$ is even, then $w_{\beta_{k}}$ is a polynomial in $\cos (r)$ whose exponents are even, therefore $w_{\beta_{k}}$ is symmetric with respect to $\frac{\pi}{2}$ which implies $w_{\beta_{k}}(\pi)=w_{\beta_{k}}(0)=1$. If $k$ is odd, then $w_{\beta_{k}}$ is a polynomial in $\cos (r)$ whose exponents are odd, then $w_{\beta_{k}}$ is antisymmetric with respect to $\frac{\pi}{2}$ and we have $w_{\beta_{k}}(\pi)=-1$. By the previous comment, when we move from $k$ to $k+1$, the corresponding solutions change sign in $\pi$, it follows that $w_{\beta_{k+1}}$ must have at least one more zero than $w_{\beta_{k}}$. Therefore, by induction $w_{\beta_{k+1}}$ has at least $k+1$ zeroes in $(0, \pi)$. By the previous comments we conclude that for any positive integer $k, w_{\beta_{k}}$ has exactly $k$ zeroes in $(0, \pi)$ which are simple, as claimed.

Let $C$ be the closure of the family of positive nontrivial solutions $(u, \lambda)$ of $S(u, \lambda)=$ 0 in $C^{2, \alpha}\left(X_{I}\right)$. Let $C_{k}$ be the connected component of $C$ containing the bifurcation point $\left(1, \lambda_{k}\right)$. For the curve of nontrivial solutions $(u(t), \lambda(t))$ close to $\left(1, \lambda_{k}\right)$ we have $u(t)=1+t w_{\beta_{k}}+o\left(t^{2}\right)$. It follows then from the previous comments that $(u(t), \lambda(t)) \in C_{k}$ and if $u \neq 1$ then $u$ has exactly $k$-zeroes in $(0, \pi)$. In particular $\left(1, \lambda_{i}\right)$ does not belong to $C_{k}$ if $i \neq k$.

\section{Claim 1: $C_{k}$ is not compact}

Proof. To prove the claim we will use the global bifurcation theorem of Rabinowitz, as in (for instance) [1, Theorem 4.8]. Let us briefly describe how to write the equation in the setup of the global bifurcation theorem.

Given a solution $u: \mathbf{S}^{n} \times \mathbf{S}^{n} \rightarrow \mathbb{R}_{>0}$ of equation (2) we let $w=u-1$. Then, $u$ is a solution of (2) if and only if $w$ verifies

$$
-\Delta_{G_{\delta}} w+\lambda(w+1)=\lambda(w+1)^{p-1} .
$$

Let $K: C^{2, \alpha}\left(X_{I}\right) \rightarrow C^{2, \alpha}\left(X_{I}\right)$ be the inverse operator of

$$
-\Delta_{G_{\delta}}+I d: C^{4, \alpha}\left(X_{I}\right) \rightarrow C^{2, \alpha}\left(X_{I}\right) .
$$

The operator $K$ is linear and compact. Consider the region

$$
D:=\left\{(w, \eta) \in C^{2, \alpha}\left(X_{I}\right) \times \mathbb{R}: w>-1, \eta>1\right\},
$$

and define $T: D \rightarrow C^{2, \alpha}\left(X_{I}\right)$ by

$$
\left.T(w, \eta)=\frac{\eta-1}{p-2} K\left((w+1)^{p-1}-(p-1) w-1\right)\right) .
$$

$T$ is a compact operator and for each $\eta>1, T(0, \eta)=0, \quad T_{w}^{\prime}(0, \eta)=0$. Now define $F: D \rightarrow C^{2, \alpha}\left(X_{I}\right)$ by

$$
F(w, \eta)=w-\eta K(w)-T(w, \eta)
$$


Note that $F(0, \eta)=0$ for each $\eta$. And if we apply $-\Delta_{G_{\delta}}+I d$ to the equation $F(w, \eta)=0$, we see that $F(w, \eta)=0$ if and only if

$$
-\Delta_{G_{\delta}} w-\frac{\eta-1}{p-2}\left((w+1)^{p-1}-(w+1)\right)=0
$$

Therefore, $F(w, \eta)=0$ if and only if $w$ is a solution of equation (9) for $\lambda=\frac{\eta-1}{p-2}$.

Let $\eta_{k}=\lambda_{k}(p-2)+1$. Similarly as before we let $B$ be the closure of the nontrivial solutions $(w, \eta)$ of $F(w, \eta)=0$ in $D$ and $B_{k}$ be the connected component of $B$ containing the bifurcation point $\left(0, \eta_{k}\right)$. In this context we can apply the global bifurcation theorem of Rabinowitz ([1], Theorem 4.8): it follows that either $B_{k}$ is not compact or $B_{k}$ contains another bifurcation point $\left(0, \eta_{j}\right)$ with $j \neq k$. But we have seen that the second condition does not hold, therefore $B_{k}$ is not compact. But $C_{k}=\left\{\left(w+1, \frac{\eta-1}{p-2}\right):(w, \eta) \in B_{k}\right\}$ and therefore $C_{k}$ is not compact.

Note that $\left(\mathbf{S}^{n} \times \mathbf{S}^{n}, G_{\delta}\right)$ has positive Ricci curvature and by [7, Theorem 6.1 ], there exist $\rho>0$ such that if $\lambda<\rho$ the equation (2) only has the trivial solution.

Claim 2: For any $\lambda_{0}, 0<\rho<\lambda_{0}$, the set

$$
A:=\left\{(u, \lambda): S(u, \lambda)=0, \lambda \in\left[\rho, \lambda_{0}\right]\right\},
$$

is compact.

The claim is well-known, see for instance the proof of [19, Lemma 2.2]. First one has to note that there exists $\Lambda>0$ such that if $(u, \lambda) \in A$ then $u \leq \Lambda$. This is proved by the blow up technique (see for instance the proof in [27, Theorem 2.1, page 200]): if there exists a sequence $\left(u_{i}, \lambda_{i}\right) \in A$ and $x_{i} \in \mathbf{S}^{n} \times \mathbf{S}^{n}$ such that $u_{i}\left(x_{i}\right) \rightarrow \infty$ then by taking a subsequence we can assume that $x_{i} \rightarrow x \in \mathbf{S}^{n} \times \mathbf{S}^{n}$ and $\lambda_{i} \rightarrow \lambda \in\left[\rho, \lambda_{0}\right]$. Then by taking a normal neighborhood of $x$ and renormalizing $u_{i}$ one would construct as a limit a positive solution of $\Delta u+\lambda u^{p-1}=0$ in $\mathbb{R}^{2 n}$. But since $p<p_{2 n}$ is subcritical such solution does not exist by [11]. Then we consider again the compact operator $K: C^{2, \alpha}\left(X_{I}\right) \rightarrow C^{2, \alpha}\left(X_{I}\right)$ from the proof of Claim 1 (the inverse of $-\Delta_{G_{\delta}}+I d$ ) and point out that $S(u, \lambda)=0$ if and only if $u=K\left(\lambda u^{p-1}-(\lambda-1) u\right)$ : this implies that $A$ is compact.

If there exists $\lambda_{*}>\lambda_{k}$ such that it does not exist $u \neq 0$ such that $\left(u, \lambda_{*}\right) \in C_{k}$, then since $C_{k}$ is connected we have that $C_{k} \subset C_{+}^{2, \alpha}\left(X_{I}\right) \times\left[\rho, \lambda_{*}\right]$. But then Claim 2 would imply that $C_{k}$ is compact, contradicting Claim 1. Then for any $\lambda>\lambda_{k}$ there exist $u \neq 0$ such that $(u, \lambda) \in C_{k}$. Since $C_{k} \cap C_{j}=\emptyset$ if $j \neq k$, this proves Theorem 2.2 .

\section{ReFERENCES}

[1] A. Ambrosetti and A. Malchiodi, Nonlinear analysis and semilinear elliptic problems, Cambridge University Press. 2007 
[2] K. Akutagawa, L. Florit, J. Petean, On Yamabe constants of Riemannian products, Comm. Anal. Geom. 15 (2007), 947-969.

[3] B. Ammann, M. Dahl, E. Humbert, Smooth Yamabe invariant and surgery, J. Differential Geometry 94 (2013), 1-58.

[4] B. Ammann, E. Humbert, The second Yamabe invariant, J. Funct. Anal. 235 (2006), 377-412.

[5] T. Aubin, Equations differentielles non-lineaires et probleme de Yamabe concernant la courbure scalaire, J. Math. Pures Appl. 55 (1976), 269-296.

[6] R. Bettiol, P. Piccione, Multiplicity of solutions to the Yamabe problem on collapsing Riemannian submersions, Pacific J. Math. 266, 1-21 (2013).

[7] M. F. Bidaut-Véron and L. Véron, Nonlinear elliptic equations on compact Riemann- ian manifolds and asymptotics of Emden equations, Invent. Math., 106 (1991), 489-539.

[8] M. Clapp, J. Faya, A. Pistoia Nonexistence and multiplicity of solutions to elliptic problems with supercritical exponents. Calc. Var. Partial Differ. Equ. 48 (2013), 611-623

[9] M. Clapp, J.C. Fernández. Multiplicity of nodal solution to the Yamabe problem. Calc. Var. Partial Differ. Equ. 56:145 (2017), 611-623

[10] E. N. Dancer, A. M. Micheletti, A. Pistoia, Multipeak solutions for some singularly perturbed nonlinear elliptic problems on Riemannian manifolds, Manuscripta Math. 128 (2009), 163-193.

[11] B. Gidas and J. Spruck. Global and local behavior of positive solutions of nonlinear elliptic equations Comm. Pure Appl. Math. 34, 1981, pp. 525-598.

[12] L.L. de Lima, P. Piccione, M. Zedda, On bifurcation of solutions of the Yamabe problem in product manifolds, Annales de L'institute Henri Poincare (C) Non Linear Analysis 29, 261-277 (2012).

[13] S. Deng, Z. Khemiri and F. Mahmoudi, On spike solutions for a singularly perturbed problem in a compact Riemannian manifold.

[14] Y. Ding. On a conformally invariant elliptic equation on $\mathbb{R}^{n}$. Comm. Math. Phys. 107 (1986), no. $2,331-335$.

[15] J. C. Fernandez and J. Petean, Low energy nodal solutions to the Yamabe equation, arXiv:1807.06114

[16] G. Henry, F. Madani, Equivariant Second Yamabe constant, arXiv:1612.03119, to appear in The Journal of Geometruc Analysis.

[17] G. Henry, J. Petean, Isoparametric hypersurfaces and metrics of constant scalar curvature, Asian J. Math. 18, 53-68 (2014).

[18] E.L. Ince Ordinary Differential Equations . Dover, New York, 1956.

[19] Q. Jin, Y. Y. Li, H. Xu, Symmetry and asymetry: the method of moving spheres, Advances in Differential Equations 13 (2008), 601-640.

[20] A. M. Micheletti, A. Pistoia, The role of the scalar curvature in a nonlinear elliptic problem on Riemannian manifolds, Calc. Var. 34 (2009), 233-265.

[21] L. Nirenberg, Topics in nonlinear functional analysis, New York University Lecture Notes, New York, 1974.

[22] M. Obata, The conjectures on conformal transformations of Riemannian manifolds, J. Diff. Geom., No. 6, 247-258, 1971.

[23] J. Petean, Metrics of constant scalar curvature conformal to Riemannian products, Proc. Amer. Math. Soc. 138, 2897-2905 (2010).

[24] F. Robert, J. Vétois. Sign-Changing Blow-Up for Scalar Curvature Type Equations. Comm. Partial Differential Equations 38 (2013), 1437-1465.

[25] R. Schoen, Conformal deformation of a Riemannian metric to constant scalar curvature, J. Differential Geometry 20 (1984), 479-495.

[26] R. Schoen, Variational theory for the total scalar curvature functional for Riemannian metrics and related topics, Lecture Notes in Math. 1365, Springer-Verlag, Berlin, 1989, 120-154.

[27] R. Schoen and S. T. Yau, Lectures on Differential Geometry. International Press Somerville, Massachusetts, U.S.A. 2010. 
[28] N. Trudinger, Remarks concerning the conformal deformation of Riemannian structures on compact manifolds, Ann. Scuola Norm. Sup. Pisa (3) 22 (1968), 265274.

[29] H. Yamabe, On a deformation of Riemannian structures on compact manifolds, Osaka Math. J. 12 (1960), 21-37.

[30] Q. M. Wang, Isoparametric functions on Riemannian manifolds I, Math. Ann. 277 (1987), 639-646.

Centro de Investigación en Matemáticas, Cimat, Calle Jalisco s/n, 36023 Guanajuato, Guanajuato, México

E-mail address: jimmy@cimat.mx

Centro de Investigación en Matemáticas, Cimat, Calle Jalisco s/n, 36023 Guanajuato, Guanajuato, México. Universidad de Costa Rica. Sede de Occidente. 20201. Alajuela. Costa Rica.

E-mail address: hector.barrantes@cimat.mx, hector.barrantes@ucr.ac.cr 